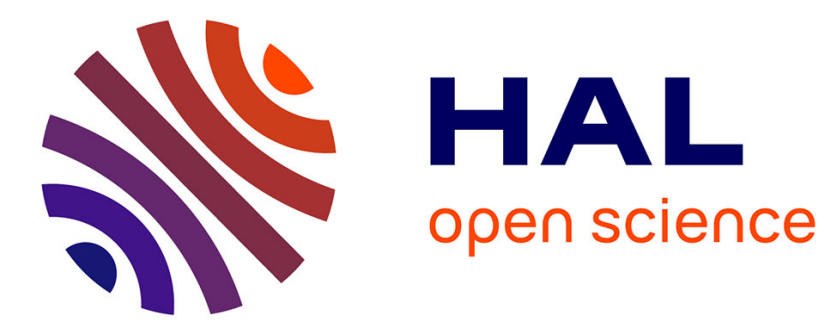

\title{
Private versus public consumption within groups: testing the nature of goods from aggregate data
}

Laurens Cherchye, Bram de Rock, Vincenzo Platino

\section{To cite this version:}

Laurens Cherchye, Bram de Rock, Vincenzo Platino. Private versus public consumption within groups : testing the nature of goods from aggregate data. 2010. halshs-00543999

\section{HAL Id: halshs-00543999 \\ https://shs.hal.science/halshs-00543999}

Submitted on 7 Dec 2010

HAL is a multi-disciplinary open access archive for the deposit and dissemination of scientific research documents, whether they are published or not. The documents may come from teaching and research institutions in France or abroad, or from public or private research centers.
L'archive ouverte pluridisciplinaire HAL, est destinée au dépôt et à la diffusion de documents scientifiques de niveau recherche, publiés ou non, émanant des établissements d'enseignement et de recherche français ou étrangers, des laboratoires publics ou privés. 


\section{Documents de Travail du Centre d'Economie de la Sorbonne}

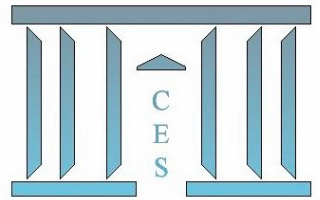

Private versus public consumption within groups : testing the nature of goods from aggregate data

Laurens Cherchye, Bram De Rock, Vincenzo Platino

2010.90

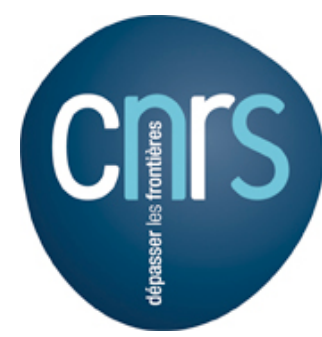




\title{
Private versus public consumption within groups: testing the nature of goods from aggregate data*
}

\author{
Laurens Cherchyeł Bram De Rock ${ }^{\ddagger}$ and Vincenzo Platino ${ }^{\S}$
}

August, 2010

\begin{abstract}
We study the testability implications of public versus private consumption in collective models of group consumption. The distinguishing feature of our approach is that we start from a revealed preference characterization of collectively rational behavior. Remarkably, we find that assumptions regarding the public or private nature of specific goods do have testability implications, even if one only observes the aggregate group consumption. In fact, these testability implications apply as soon as the analysis includes three goods and four observations. This stands in sharp contrast with existing results that start from a differential characterization of collectively rational behavior. In our opinion, our revealed preference approach obtains stronger testability conclusions because it focuses on a global characterization of collective rationality, whereas the differential approach starts from a local characterization.
\end{abstract}

JEL Classification: D11, D12, D13, C14.

Keywords: multi-person group consumption, collective model, revealed preferences, public goods, private goods, consumption externalities.

${ }^{*}$ We thank Georg Kirchsteiger and Paola Conconi for useful comments.

${ }^{\dagger}$ CentER, Tilburg University, and Center for Economic Studies, University of Leuven. E. Sabbelaan 53, B-8500 Kortrijk, Belgium. E-mail: laurens.cherchye@kuleuven-kortrijk.be. Laurens Cherchye gratefully acknowledges financial support from the Research Fund K.U.Leuven through the grant STRT1/08/004.

${ }^{\ddagger}$ ECARES and ECORE, Université Libre de Bruxelles. Avenue F.D. Roosevelt 50, 1050 Brussels, Belgium. E-mail: bderock@ulb.ac.be.

${ }^{\S}$ Paris School of Economics-Université Paris 1 Panthéon-Sorbonne, Centre d'Economie de la Sorbonne, 106-112 Boulevard de l'Hôpital, 75647 Paris Cedex 13, France. E-mail: Vincenzo.Platino@malix.univ-paris1.fr. 


\section{Introduction}

There is a growing consensus that multi-person group (e.g. household) consumption behavior should no longer be treated as if the group were a single decision maker that optimizes a group utility function subject to the group budget constraint. Indeed, this so-called unitary model of group consumption imposes empirically testable restrictions on the group demand function (e.g. Slutsky symmetry) that are frequently rejected when confronted with data on multi-person group consumption. See, for example, Browning and Chiappori (1998) and references therein.

Because of these empirical problems of the unitary model, the collective model has become increasingly popular to analyze group consumption behavior. Chiappori (1988, 1992) originally introduced this model for describing household labor supply behavior when all consumption is private and consumption externalities are absent (i.e. individual preferences are egoistic). More recently, Browning and Chiappori (1998) suggested a most general collective consumption model, which does account for public consumption in addition to private consumption. This model also allows for externalities related to privately consumed quantities. In addition, Browning and Chiappori make the minimalistic assumption that the empirical analyst only observes the aggregate consumption and does not know which part of the quantities are publicly or privately consumed. Focusing on a 'differential' characterization of this general model, they establish that for two-person groups collectively rational group behavior requires a pseudo-Slutsky matrix that can be written as the sum of a symmetric negative semi-definite matrix and a rank one matrix. ${ }^{1}$

Building further on the original work of Browning and Chiappori (1998), Chiappori and Ekeland (2006) particularly focused on the testability conclusions regarding the private and public nature of group consumption. Their main conclusion is that, following a differential approach, "the private or public nature of consumption within the group is not testable from aggregate data on group behavior". More specifically, they show that, when only observing the aggregate group consumption, the general collective consumption model has exactly the same testability implications as two more specific collective models, i.e. a first benchmark model that assumes all consumption is public and a second benchmark model that assumes all consumption is

\footnotetext{
${ }^{1}$ The term differential refers to the fact that the characterization is obtained by integrating and/or differentiating the functional specifications of the fundamentals (e.g. the individual preferences of the group members) of the model.
} 
private and preferences are egoistic (i.e. no consumption externalities).

In this paper, we complement the results of Chiappori and Ekeland. In the tradition of Afriat (1967) and Varian (1982), ${ }^{2}$ we investigate the same testability questions by focusing on the 'revealed preference' characterization of the collective consumption model. Such a revealed preference characterization does not rely on any functional specification regarding the group consumption process; it typically focuses on revealed preference axioms that summarize the empirical implications of theoretical consumption models. Our study extends earlier work of Cherchye, De Rock and Vermeulen (2007, 2010a, 2010b), who developed the revealed preference characterization of the (general and specific) collective consumption models mentioned above.

Remarkably, in contrast to the findings for the differential approach, we will conclude that our revealed preference approach does imply testability of privateness versus publicness of consumption, even if one only observes the aggregate group consumption. In addition, we will obtain that the model with all consumption public is independent from (or non-nested with) the model that assumes all consumption is private and preferences are egoistic: a data set that satisfies the revealed preference conditions for the first model does not necessarily satisfy the conditions for the second model, and vice versa.

How can we interpret this difference between the testability conclusions of our approach and the ones of the differential approach? Our explanation is that Chiappori and Ekeland's differential approach focuses on 'local' conditions for collective rationality (which apply in a sufficiently small neighborhood of a given point). By contrast, the revealed preference conditions on which we focus are 'global' by construction. ${ }^{3}$ In this interpretation, the global nature of the revealed preference conditions implies stronger testability conclusions. In fact, we believe our results may have interesting implications from the viewpoint of practical applications. For example, they suggest that a practitioner may usefully apply the revealed preference characterization to verify if the data satisfies a particular specification the collective model (in terms of publicly and/or privately consumed goods), prior to the actual empirical analysis.

One final remark is in order before entering our analysis. Following a similar revealed preference approach, Cherchye, De Rock and Vermeulen (2010a) also con-

\footnotetext{
${ }^{2}$ See also Samuelson (1938), Houthakker (1950) and Diewert (1973) for seminal contributions on the revealed preference approach to analyzing consumption behavior.

${ }^{3}$ See for example Hurwicz (1971) and Pollak (1990) for discussions on the difference between the global revealed preference approach and the local differential approach.
} 
sidered testability of the private versus public nature of consumption within groups. A specific feature of their analysis is that it allowed for non-convex preferences of the individual group members. These authors obtain the same nontestability conclusion as Chiappori and Ekeland (2006). Our following analysis differs from the one of Cherchye, De Rock and Vermeulen (2010a) in that we assume that individual preferences are convex (and represented by concave utility functions); this assumption of convex preferences follows the original analysis of Chiappori and Ekeland. As indicated above, we now do obtain different testability implications under alternative assumptions on the (public or private) nature of goods. When comparing this to the findings of Cherchye, De Rock and Vermeulen (2010a), we conclude that the assumption of convex preferences is crucial for obtaining our testability conclusions.

The remainder of the paper unfolds as follows. To set the stage, Section 2 defines collectively rational group consumption behavior in terms of the (general and specific) collective models that we will consider. Section 3 discusses the revealed preference characterization of such rational behavior. Section 4 shows our testability results on public versus private consumption in the group. Section 5 summarizes our main conclusions.

\section{Collective rationality}

Following Chiappori and Ekeland (2006, 2009), we will concentrate on three collective consumption models in what follows. We will consider the general collective model (general-CR) of Browning and Chiappori (1998) as well as two specific benchmark models, i.e. the collective model with all goods public (public-CR) and the collective model with all consumption private and egoistic preferences (egoistic-CR). In this section we introduce the necessary concepts to study these three collective models.

Throughout, we consider groups (or households) that consist of two members. ${ }^{4}$ We assume a group that purchases the (non-zero) $N$-vector of quantities $\mathbf{q} \in \mathbb{R}_{+}^{N}$ with corresponding prices $\mathbf{p} \in \mathbb{R}_{++}^{N}$. All quantities can be consumed privately, publicly, or both. For the general collective model, we will assume that the empirical analyst has no information on the decomposition of the observed $\mathbf{q}$ into the bundles of private quantities $\mathbf{q}^{1}, \mathbf{q}^{2}$ and the bundle of public quantities $\mathbf{q}^{h}$. Therefore, we need to intro-

\footnotetext{
${ }^{4}$ The results below can be generalized towards the setting of $M$ members, with $M \geq 2$. However, we believe that the core arguments underlying our results are better articulated for this simple case.
} 
duce (unobserved) feasible personalized quantities $\widehat{\mathbf{q}}$ that comply with the (observed) aggregate quantities q. More formally, we define

$$
\widehat{\mathbf{q}}=\left(\mathfrak{q}^{1}, \mathfrak{q}^{2}, \mathfrak{q}^{h}\right) \text { with } \mathfrak{q}^{1}, \mathfrak{q}^{2}, \mathfrak{q}^{h} \in \mathbb{R}_{+}^{N} \text { and } \mathfrak{q}^{1}+\mathfrak{q}^{2}+\mathfrak{q}^{h}=\mathbf{q}
$$

Each $\widehat{\mathbf{q}}$ captures a feasible decomposition of the aggregate quantities $\mathbf{q}$ into private quantities and public quantities. This will be useful for modeling general preferences that depend on private consumption as well as public consumption. In the following, we consider feasible personalized quantities because we assume the minimalistic prior that only the aggregate quantity bundle $\mathbf{q}$ and not the 'true' personalized quantities are observed. Throughout, we will use that each $\widehat{\mathbf{q}}$ defines a unique $\mathbf{q}$.

The collective model explicitly recognizes the individual (convex) preferences of the group members. For the general model, these preferences may depend not only on the own private quantities and the public quantities, but also on the other individual's private quantities. This allows for externalities between the group members. Formally, this means that the preferences of each group member $m(m=1,2)$ can be represented by a well-behaved utility function of the form $U^{m}\left(\mathbf{q}^{1}, \mathbf{q}^{2}, \mathbf{q}^{h}\right)$, with $\mathbf{q}=\mathbf{q}^{1}+\mathbf{q}^{2}+\mathbf{q}^{h}$ and $m=1,2 .^{5}$

Suppose then that we observe $T$ choices of $N$-valued bundles. For each observation $t$ the vector $\mathbf{q}_{t} \in \mathbb{R}_{+}^{N}$ records the quantities chosen by the group under the prices $\mathbf{p}_{t} \in \mathbb{R}_{++}^{N}$ (with strictly positive components). We let $S=\left\{\left(\mathbf{p}_{t}, \mathbf{q}_{t}\right) ; t=1, \ldots, T\right\}$ be the corresponding set of $T$ observations. ${ }^{6}$ A collective rationalization of a set of observations $S$ requires the existence of utility functions $U^{1}$ and $U^{2}$ such that each observed quantity bundle can be characterized as Pareto efficient. Thus, we get the following definition.

Definition 1 (general-CR) Let $S=\left\{\left(\mathbf{p}_{t}, \mathbf{q}_{t}\right) ; t=1, \ldots, T\right\}$ be a set of observations. A pair of utility functions $U^{1}$ and $U^{2}$ provides a general-CR of $S$ (i.e. a collective rationalization in terms of the general collective model), if for each observation $t$ there exist feasible personalized quantities $\widehat{\mathbf{q}}_{t}$ such that $U^{m}(\widehat{\mathbf{z}})>U^{m}\left(\widehat{\mathbf{q}}_{t}\right)$ implies $U^{l}(\widehat{\mathbf{z}})<U^{l}\left(\widehat{\mathbf{q}}_{t}\right)(m \neq l)$ for all feasible personalized quantities $\widehat{\mathbf{z}}$ with $\mathbf{p}_{t} \mathbf{q}_{t} \geq \mathbf{p}_{t} \mathbf{z}$.

The two benchmark cases considered below involve restrictions on the individual

\footnotetext{
${ }^{5} \mathrm{As}$ in the differential approach, we say that a function is well-behaved if it is concave, differentiable and monotonically increasing.

${ }^{6}$ For ease of exposition, the scalar product $\mathbf{p}_{t}^{\prime} \mathbf{q}_{t}$ is written as $\mathbf{p}_{t} \mathbf{q}_{t}$.
} 
preferences and the nature of the goods. In the first case we assume that all consumption is public. We formalize this by assuming individuals preferences that are represented by a well-behaved utility function $U_{p u b}^{m}\left(\mathbf{q}^{h}\right)$. Clearly, in this case we have $\mathfrak{q}^{h}=\mathbf{q}\left(\right.$ or $\left.\mathfrak{q}^{1}+\mathfrak{q}^{2}=\mathbf{0}\right)$, i.e. the true personalized quantities are effectively observed. Given all this, Definition 1 directly leads to the following definition.

Definition 2 (public-CR) Let $S=\left\{\left(\mathbf{p}_{t}, \mathbf{q}_{t}\right) ; t=1, \ldots, T\right\}$ be a set of observations. A pair of utility functions $U_{p u b}^{1}$ and $U_{\text {pub }}^{2}$ provides a public-CR of $S$ (i.e. a collective rationalization in terms of the collective model with only public consumption), if for each observation $t$ we have that $U_{p u b}^{m}(\mathbf{z})>U_{p u b}^{m}\left(\mathbf{q}_{t}\right)$ implies $U_{p u b}^{l}(\mathbf{z})<U_{p u b}^{l}\left(\mathbf{q}_{t}\right)(m \neq$ l) for all $\mathbf{z}$ with $\mathbf{p}_{t} \mathbf{q}_{t} \geq \mathbf{p}_{t} \mathbf{z}$.

The second benchmark case assumes that all consumption is private, i.e. $\mathfrak{q}^{1}+\mathfrak{q}^{2}=$ $\mathbf{q}$ ( or $\left.\mathbf{q}^{h}=\mathbf{0}\right)$. In addition, the individuals have egoistic preferences, which implies that they only care for their own consumption (i.e. no consumption externalities). We formalize this by assuming individual preferences that are represented by a wellbehaved utility function $U_{e g o}^{m}\left(\mathbf{q}^{m}\right)$, with $m=1,2$. The corresponding concept of collective rationality is as follows.

Definition 3 (egoistic-CR) Let $S=\left\{\left(\mathbf{p}_{t}, \mathbf{q}_{t}\right) ; t=1, \ldots, T\right\}$ be a set of observations. A pair of utility functions $U_{\text {ego }}^{1}$ and $U_{\text {ego }}^{2}$ provides an egoistic-CR of $S$ (i.e. a collective rationalization in terms of the collective model with all consumption private and egoistic preferences), if for each observation $t$ there exist feasible personalized quantities $\widehat{\mathbf{q}}_{t}$, with $\mathfrak{q}^{h}=\mathbf{0}$, such that $U_{\text {ego }}^{m}(\widehat{\mathbf{z}})>U_{\text {ego }}^{m}\left(\widehat{\mathbf{q}}_{t}\right)$ implies $U_{\text {ego }}^{l}(\widehat{\mathbf{z}})<U_{\text {ego }}^{l}\left(\widehat{\mathbf{q}}_{t}\right)$ $(m \neq l)$ for all feasible personalized quantities $\widehat{\mathbf{z}}$ with $\mathbf{p}_{t} \mathbf{q}_{t} \geq \mathbf{p}_{t} \mathbf{z}$ and $\mathfrak{z}^{h}=\mathbf{0}$.

\section{Revealed preference characterization}

Cherchye, De Rock and Vermeulen (2007, 2010b) derived the revealed preference characterizations for the three models discussed in the previous section. To formally define these revealed preference conditions, we will use the concept of feasible personalized prices $\widehat{\mathbf{p}}^{1}$ and $\widehat{\mathbf{p}}^{2}$.

$$
\begin{aligned}
\widehat{\mathbf{p}}^{1} & =\left(\mathfrak{p}^{1}, \mathfrak{p}^{2}, \mathfrak{p}^{h}\right) \text { and } \widehat{\mathbf{p}}^{2}=\left(\mathbf{p}-\mathfrak{p}^{1}, \mathbf{p}-\mathfrak{p}^{2}, \mathbf{p}-\mathfrak{p}^{h}\right) \text { with } \\
\mathfrak{p}^{1}, \mathfrak{p}^{2}, \mathfrak{p}^{h} & \in \mathbb{R}_{+}^{N} \text { and } \mathfrak{p}^{c} \leq \mathbf{p} \text { for } c=1,2, h
\end{aligned}
$$


This concept complements the concept of feasible personalized quantities defined above: $\widehat{\mathbf{p}}^{1}$ and $\widehat{\mathbf{p}}^{2}$ capture the fraction of the price for the personalized quantities $\widehat{\mathbf{q}}$ that is borne by the respective members. $\mathfrak{p}^{1}$ and $\mathfrak{p}^{2}$ refer to private quantities and are used to express the willingness to pay for the externalities related to these private quantities; $\mathfrak{p}^{h}$ refers to the public quantities and are similarly used to express the willingness to pay for the public quantities.

The revealed preference conditions make use of the Generalized Axiom of Revealed Preference (GARP). Varian (1982) introduced the GARP condition for individually rational behavior for observed prices and quantities; i.e. he showed that it is a necessary and sufficient condition for the observed quantity choices to maximize a single utility function under the given budget constraint. We focus on the same condition in terms of feasible personalized prices and quantities; the next Proposition 1 will establish that collective rationality as defined in the above definitions requires GARP consistency for each individual member.

Definition 4 Consider feasible personalized prices and quantities for a set of observations $S=\left\{\left(\mathbf{p}_{t}, \mathbf{q}_{t}\right) ; t=1, \ldots, T\right\}$. For $m=1,2$, the $\operatorname{set}\left\{\left(\widehat{\mathbf{p}}_{t}^{m}, \widehat{\mathbf{q}}_{t}^{m}\right) ; t=1, \ldots, T\right\}$ satisfies GARP if there exist relations $R_{0}^{m}, R^{m}$ that meet:

(i) if $\widehat{\mathbf{p}}_{s}^{m} \widehat{\mathbf{q}}_{s} \geq \widehat{\mathbf{p}}_{s}^{m} \widehat{\mathbf{q}}_{t}$ then $\widehat{\mathbf{q}}_{s} R_{0}^{m} \widehat{\mathbf{q}}_{t}$;

(ii) if $\widehat{\mathbf{q}}_{s} R_{0}^{m} \widehat{\mathbf{q}}_{u}, \widehat{\mathbf{q}}_{u} R_{0}^{m} \widehat{\mathbf{q}}_{v}, \ldots, \widehat{\mathbf{q}}_{z} R_{0}^{m} \widehat{\mathbf{q}}_{t}$ for some (possibly empty) sequence (u,v, ..., z) then $\widehat{\mathbf{q}}_{s} R^{m} \widehat{\mathbf{q}}_{t}$;

(iii) if $\widehat{\mathbf{q}}_{s} R^{m} \widehat{\mathbf{q}}_{t}$, then $\widehat{\mathbf{p}}_{t} \widehat{\mathbf{q}}_{t} \leq \widehat{\mathbf{p}}_{t} \widehat{\mathbf{q}}_{s}$.

We can now state the revealed preference characterization of the general collective model (i.e. general-CR) that is derived in Cherchye, De Rock and Vermeulen (2007).

Proposition 1 Let $S=\left\{\left(\mathbf{p}_{t}, \mathbf{q}_{t}\right) ; t=1, \ldots, T\right\}$ be a set of observations. The following conditions are equivalent:

(i) there exists a combination of well-behaved utility functions $U^{1}$ and $U^{2}$ that provide a general-CR of $S$;

(ii) there exist feasible personalized prices and quantities such that for each member $m=1,2$, the set $\left\{\left(\widehat{\mathbf{p}}_{t}^{m}, \widehat{\mathbf{q}}_{t}\right) ; t=1, \ldots, T\right\}$ satisfies GARP.

Essentially, condition (ii) states that collective rationality requires individual rationality (i.e. GARP consistency) of each member in terms of personalized prices 
and quantities. In general, however, the true personalized prices and quantities are unobserved. Therefore, it is only required that there must exist at least one set of feasible personalized prices and quantities that satisfies the condition.

The characterization in Proposition 1 is easily adapted to the two benchmark cases considered in the previous section; see also Cherchye, De Rock and Vermeulen (2010b) for more discussion. For a public-CR of the data we need to include that all consumption is public. The implication is that only the willingness to pay for the public consumption will be relevant for the GARP test. This is contained in the following result.

Proposition 2 Let $S=\left\{\left(\mathbf{p}_{t}, \mathbf{q}_{t}\right) ; t=1, \ldots, T\right\}$ be a set of observations. The following conditions are equivalent:

(i) there exists a combination of well-behaved utility functions $U_{p u b}^{1}$ and $U_{p u b}^{2}$ that provide a public-CR of $S$;

(ii) there exist feasible personalized prices and quantities, with $\mathfrak{q}_{t}^{1}=\mathfrak{q}_{t}^{2}=\mathbf{0}$, such that for each member $m=1,2$, the set $\left\{\left(\widehat{\mathbf{p}}_{t}^{m}, \widehat{\mathbf{q}}_{t}\right) ; t=1, \ldots, T\right\}$ satisfies GARP.

Similarly, for an egoistic-CR of the data we need to add to the second condition that all consumption is private (i.e. $\mathfrak{q}_{t}^{h}=\mathbf{0}$ ) and that the preferences are egoistic, implying that the willingness to pay for externalities is zero (i.e. $\mathfrak{p}_{t}^{1}=\mathbf{p}_{t}$ and $\mathfrak{p}_{t}^{2}=\mathbf{0}$ ).

Proposition 3 Let $S=\left\{\left(\mathbf{p}_{t}, \mathbf{q}_{t}\right) ; t=1, \ldots, T\right\}$ be a set of observations. The following conditions are equivalent:

(i) there exists a combination of well-behaved utility functions $U_{\text {ego }}^{1}$ and $U_{\text {ego }}^{2}$ that provide an egoistic-CR of $S$;

(ii) there exist feasible personalized prices, with $\mathfrak{p}_{t}^{1}=\mathbf{p}_{t}$ and $\mathfrak{p}_{t}^{2}=\mathbf{0}$, and feasible personalized quantities, with $\mathfrak{q}_{t}^{h}=\mathbf{0}$, such that for each member $m=1,2$, the set $\left\{\left(\widehat{\mathbf{p}}_{t}^{m}, \widehat{\mathbf{q}}_{t}\right) ; t=1, \ldots, T\right\}$ satisfies GARP.

\section{Testing the nature of goods}

We next show that the nature of goods is testable, even if we only observe the aggregate group behavior. More specifically, we will prove two main results by means of example data sets. Firstly, we provide data sets for which there exists a general-CR 
but not a public-CR or, respectively, an egoistic-CR. This implies that consistency with the general model does not necessarily imply consistency with any of the specific benchmark models. Putting it differently, rejection of the specific benchmark models in these examples is caused by the corresponding assumptions on the nature of the goods and not by the Pareto efficiency assumption as such. Secondly, our example data sets will show that the two benchmark models are independent from (or nonnested with) each other, i.e. data consistency with one benchmark model does not necessarily imply data consistency with the other benchmark model.

\subsection{General-CR does not imply public-CR}

The following example contains a data set for which there exists a general-CR but not a public-CR. The Appendix proves our claims in the examples.

Example 1 Suppose that the dataset $S$ contains the following 3 observations of bundles consisting of 3 quantities:

$$
\begin{aligned}
& \mathbf{q}_{1}=(5,2,2)^{\prime}, \mathbf{q}_{2}=(2,5,2)^{\prime}, \mathbf{q}_{3}=(2,2,5)^{\prime} ; \\
& \mathbf{p}_{1}=(4,1,1)^{\prime}, \mathbf{p}_{2}=(1,4,1)^{\prime}, \mathbf{p}_{3}=(1,1,4)^{\prime}
\end{aligned}
$$

This dataset $S$ satisfies the conditions in Proposition 1 (i.e. there exists a generalCR), but it rejects the conditions in Proposition 2 (i.e. there does not exist a public$C R)$.

This example has two important implications. Firstly, as discussed in the introduction, it contrasts with the results of Chiappori and Ekeland (2006): following a (local) differential approach, these authors show that the general collective model and the collective model with only public consumption are indistinguishable if one only observes aggregate group behavior. Example 1 illustrates that this is no longer the case if one adopts the (global) revealed preference approach.

Secondly, the example demonstrates that we need only three goods and three observations to obtain our conclusion. In fact, these numbers provide absolute lower bounds on the number of goods and observations for the collective models to have testable implications. Indeed, it can be verified that the conditions in Propositions 1 and 2 cannot be rejected if the number of observations or the number of goods is 
smaller than three. ${ }^{7}$ Thus, as soon as collective rationality can be rejected, we can distinguish the specific model with all consumption public from the general collective consumption model. In this respect, it is also worth noting that the differential approach needs at least five goods for verifying the testable implications of the collective consumption model characterized in Propositions 2; see Browning and Chiappori (1998) and Chiappori and Ekeland (2006). The fact that our revealed preference approach requires a smaller number of goods illustrates once more that the (global) revealed preference approach can yield stronger testability conclusions than the (local) differential approach.

\subsection{General-CR does not imply egoistic-CR}

We next provide an example with a data set for which there exists a general-CR but not an egoistic-CR.

Example 2 Suppose that the dataset $S$ contains the following 4 observations of bundles consisting of 4 quantities:

$$
\begin{aligned}
& \mathbf{q}_{1}=(1,0,0,0)^{\prime}, \mathbf{q}_{2}=(0,1,0,0)^{\prime}, \mathbf{q}_{3}=(0,0,1,0)^{\prime}, \mathbf{q}_{4}=(0,0,0,1)^{\prime} ; \\
& \mathbf{p}_{1}=(7,4,4,4)^{\prime}, \mathbf{p}_{2}=(4,7,4,4)^{\prime}, \mathbf{p}_{3}=(4,4,7,4)^{\prime}, \mathbf{p}_{4}=(4,4,4,7)^{\prime}
\end{aligned}
$$

This dataset $S$ satisfies the conditions in Proposition 1 (i.e. there exists a general$C R$ ), but it rejects the conditions in Proposition 3 (i.e. there does not exist an egoistic$C R)$.

Two remarks are in order. Similar to before, we conclude that the general collective model and the model with only private consumption and egoistic preferences are distinguishable from each other. Inter alia, this implies that the private nature of the goods is testable. Again, this conclusion contrasts with the one for the differential approach. Next, for mathematical elegance we have used four goods in Example 2. Similar (but less elegant) examples exist for data sets that only consider three goods.

\footnotetext{
${ }^{7}$ If $T=2$, one can easily verify that $\mathfrak{p}_{1}^{h}=\mathbf{p}_{1}$ and $\mathfrak{p}_{2}^{h}=\mathbf{0}$ is a solution for the GARP conditions in Proposition 2 (and thus a fortiori also for the GARP conditions in Proposition 1). Next, if $N=2$, one can again verify that member 1 paying for the first good (i.e. $\left.\left(\mathfrak{p}_{t}^{h}\right)_{1}=\left(\mathbf{p}_{1}\right)_{1}\right)$ for all observations $t$ and, similarly, member 2 paying for the second good (i.e. $\left(\mathfrak{p}_{t}^{h}\right)_{2}=0$ ) for all observations $t$ obtains a solution for the GARP conditions in Proposition 2.

${ }^{8} \mathrm{~A}$ similar qualification applies to the use of zeroes in Example 2.
} 
A final observation applies to the number of observations in Example 2. We have now used four observations, which contrasts with Example 1. In fact, in general we need minimally four observations for the collective model with private goods and egoistic preferences to be distinguishable from the general collective model. This result is formalized in the following proposition, which we prove in the Appendix.

Proposition 4 Let $S=\left\{\left(\mathbf{p}_{t}, \mathbf{q}_{t}\right) ; t=1,2,3\right\}$ be a set of three observations. Suppose that there exists a general-CR of $S$, then there also exists an egoistic-CR of $S$.

\subsection{Independence of egoistic-CR and public-CR}

So far, we have shown that the general collective model is distinguishable from the two specific benchmark models. In the Appendix we argue that a similar conclusion also holds for the two benchmark cases. More precisely, we show that there exists an egoistic-CR for the data set considered in Example 1 and a public-CR for the data set considered in Example 2. Generally, this obtains that data consistency with one benchmark model does not necessarily imply data consistency with the other benchmark model.

Another interesting implication of this result is that we need no more than four observations and three goods to distinguish between the three collective consumption models under study. This conclusion directly carries over to 'intermediate' collective models that are situated between the two benchmark cases, i.e models which assume that part of the goods is privately consumed (without externalities) while all other goods are publicly consumed. See Cherchye, De Rock and Vermeulen (2010b) for a detailed discussion (including revealed preference characterizations) of these intermediate models.

\section{Conclusions}

We have shown that the revealed preference approach implies different testability conclusions for collective consumption models with alternative assumptions on the (public or private) nature of goods. In particular, we obtain different testable implications as soon as we have three goods and four observations. Interestingly, these conclusions stand in sharp contrast with the existing results for the differential approach. As indicated before, our explanation is that we focus on revealed conditions 
that are global in nature, whereas the differential approach focuses on local testability conditions. As for practical applications, our results suggest that the practitioner may fruitfully apply revealed preference conditions to verify if the data satisfies a particular specification of the collective model that (s)he wants to use in the empirical analysis.

\section{Appendix}

\section{Example 1}

There exists a general-CR of $S$. Consider the following personalized quantities and prices:

$$
\begin{aligned}
& \widehat{\mathbf{q}}_{1}=\left(\mathbf{q}_{1}, \mathbf{0}, \mathbf{0}\right), \widehat{\mathbf{q}}_{2}=\left(\frac{1}{2} \mathbf{q}_{2}, \frac{1}{2} \mathbf{q}_{2}, \mathbf{0}\right), \widehat{\mathbf{q}}_{3}=\left(\mathbf{0}, \mathbf{q}_{3}, \mathbf{0}\right) \\
& \mathfrak{p}_{t}^{1}=\mathbf{p}_{1}, \mathfrak{p}_{t}^{2}=\mathbf{0} \text { for } t=1,2,3 .
\end{aligned}
$$

Then one can easily verify that the GARP conditions in Proposition 1 are satisfied for both members. This implies that there exists a general-CR of $S$.

There exists an egoistic- $C R$ of $S$. By Proposition 3 we can conclude that the above construction also shows that there exists an egoistic-CR of $S$.

There does not exist a public- $C R$ of $S$. Let us prove this ad absurdum and assume that we have a construction of feasible prices that satisfy condition (ii) in Proposition 2.

Observe that for the given set of observations we have for any $t, s \in\{1,2,3\}$, with $t \neq s$, that $\mathbf{p}_{t} \mathbf{q}_{t}>\mathbf{p}_{t} \mathbf{q}_{s}$. Therefore we must have for our solution of feasible prices that either $\mathfrak{p}_{t}^{h} \mathbf{q}_{t}>\mathfrak{p}_{t}^{h} \mathbf{q}_{s}$ or $\left(\mathbf{p}_{t}-\mathfrak{p}_{t}^{h}\right) \mathbf{q}_{t}>\left(\mathbf{p}_{t}-\mathfrak{p}_{t}^{h}\right) \mathbf{q}_{s}$. As a result the GARP conditions in Proposition 2 require that if $\mathfrak{p}_{t}^{h} \mathbf{q}_{t} \geq \mathfrak{p}_{t}^{h} \mathbf{q}_{s}$, we must have that $\mathfrak{p}_{s}^{h} \mathbf{q}_{s} \leq \mathfrak{p}_{s}^{h} \mathbf{q}_{t}$ and thus $\left(\mathbf{p}_{s}-\mathfrak{p}_{s}^{h}\right) \mathbf{q}_{s}>\left(\mathbf{p}_{s}-\mathfrak{p}_{s}^{h}\right) \mathbf{q}_{t}$. Or, alternatively, if $\widehat{\mathbf{q}}_{t} R_{0}^{1} \widehat{\mathbf{q}}_{s}$, then we must have $\widehat{\mathbf{q}}_{s} R_{0}^{2} \widehat{\mathbf{q}}_{t}$. Given that this holds for any $t, s \in\{1,2,3\}$, with $t \neq s$, we may therefore conclude that, without losing generality, the solution of feasible prices leads to (i) $\widehat{\mathbf{q}}_{1} R_{0}^{1} \widehat{\mathbf{q}}_{2}$ and $\widehat{\mathbf{q}}_{2} R_{0}^{1} \widehat{\mathbf{q}}_{3}$ for member 1 ; and (ii) $\widehat{\mathbf{q}}_{3} R_{0}^{2} \widehat{\mathbf{q}}_{2}$ and $\widehat{\mathbf{q}}_{2} R_{0}^{2} \widehat{\mathbf{q}}_{1}$ for member 2 .

Assume that $\mathfrak{p}_{2}^{h}=\left(\pi_{1}, \pi_{2}, \pi_{3}\right)^{\prime}$. The GARP condition for member 1 in Proposition 
2 requires that

$$
\begin{aligned}
\mathfrak{p}_{2}^{h} \mathbf{q}_{2} \leq \mathfrak{p}_{2}^{h} \mathbf{q}_{1} & \Leftrightarrow 2 \pi_{1}+5 \pi_{2}+2 \pi_{3} \leq 5 \pi_{1}+2 \pi_{2}+2 \pi_{3} \\
& \Leftrightarrow 0 \leq \pi_{1}-\pi_{2} .
\end{aligned}
$$

The GARP condition for member 2 in Proposition 2 requires that

$$
\begin{aligned}
\left(\mathbf{p}_{2}-\mathfrak{p}_{2}^{h}\right) \mathbf{q}_{2} \leq\left(\mathbf{p}_{2}-\mathfrak{p}_{2}^{h}\right) \mathbf{q}_{3} \Leftrightarrow & 2\left(1-\pi_{1}\right)+5\left(4-\pi_{2}\right)+2\left(1-\pi_{3}\right) \\
& \leq 2\left(1-\pi_{1}\right)+2\left(4-\pi_{2}\right)+5\left(1-\pi_{3}\right) \\
\Leftrightarrow & 3 \leq \pi_{2}-\pi_{3} .
\end{aligned}
$$

Together this implies that $3 \leq \pi_{2} \leq \pi_{1}$, which gives us the wanted contradiction since by construction $\pi_{1} \leq 1$. We thus conclude that there cannot exists a public-CR of the data set in Example 1.

\section{Example 2}

There exists a general-CR of $S$. Consider the following personalized quantities and prices:

$$
\begin{aligned}
& \widehat{\mathbf{q}}_{1}=\left(\mathbf{0}, \mathbf{0}, \mathbf{q}_{1}\right), \widehat{\mathbf{q}}_{2}=\left(\mathbf{0}, \mathbf{0}, \mathbf{q}_{2}\right), \widehat{\mathbf{q}}_{3}=\left(\mathbf{0}, \mathbf{0}, \mathbf{q}_{3}\right), \widehat{\mathbf{q}}_{4}=\left(\mathbf{0}, \mathbf{0}, \mathbf{q}_{4}\right) ; \\
& \mathfrak{p}_{1}^{h}=(6,2,2,2)^{\prime}, \mathfrak{p}_{2}^{h}=(4,3.5,0,0)^{\prime}, \mathfrak{p}_{3}^{h}=(4,4,3.5,0)^{\prime}, \mathfrak{p}_{4}^{h}=(2,2,2,1)^{\prime} .
\end{aligned}
$$

Then one can easily verify that the GARP conditions in Proposition 1 are satisfied for both members. This implies that there exists a general-CR of $S$.

There exists a public-CR of $S$. By Proposition 2 we can conclude that the above construction also shows that there exists a public-CR of $S$.

There does not exist an egoistic-CR of $S$. Let us prove this ad absurdum and assume that we have a construction of feasible prices that satisfy condition (ii) in Proposition 3.

Again we observe that for the given set of observations we have for any $t, s \in$ $\{1,2,3,4\}$, with $t \neq s$, that $\mathbf{p}_{t} \mathbf{q}_{t}>\mathbf{p}_{t} \mathbf{q}_{s}$. Therefore, without losing generality, we can as before assume that the solution of feasible prices leads to (i) $\widehat{\mathbf{q}}_{1} R_{0}^{1} \widehat{\mathbf{q}}_{2}, \widehat{\mathbf{q}}_{2} R_{0}^{1} \widehat{\mathbf{q}}_{3}$ and 
$\widehat{\mathbf{q}}_{3} R_{0}^{1} \widehat{\mathbf{q}}_{4}$ for member 1 ; and (ii) $\widehat{\mathbf{q}}_{4} R_{0}^{2} \widehat{\mathbf{q}}_{3}, \widehat{\mathbf{q}}_{3} R_{0}^{1} \widehat{\mathbf{q}}_{2}$ and $\widehat{\mathbf{q}}_{2} R_{0}^{2} \widehat{\mathbf{q}}_{1}$ for member 2 .

Assume that $\mathfrak{q}_{2}^{1}=(0, \alpha, 0,0)$ and $\mathfrak{q}_{3}^{1}=(0,0, \beta, 0)$. The GARP conditions for the two members in Proposition 3 require that the following holds:

$$
\begin{aligned}
& \widehat{\mathbf{p}}_{2}^{1} \widehat{\mathbf{q}}_{2} \leq \widehat{\mathbf{p}}_{2}^{1} \widehat{\mathbf{q}}_{1} \quad \Leftrightarrow \quad 7 \alpha \leq 4 ; \\
& \widehat{\mathbf{p}}_{3}^{1} \widehat{\mathbf{q}}_{3} \leq \widehat{\mathbf{p}}_{3}^{1} \widehat{\mathbf{q}}_{2} \quad \Leftrightarrow \quad 7 \beta \leq 4 \alpha \leq 4 ; \\
& \widehat{\mathbf{p}}_{2}^{2} \widehat{\mathbf{q}}_{2} \leq \widehat{\mathbf{p}}_{2}^{2} \widehat{\mathbf{q}}_{3} \quad \Leftrightarrow \quad 7(1-\alpha) \leq 4(1-\beta) \leq 4 ; \\
& \widehat{\mathbf{p}}_{3}^{2} \widehat{\mathbf{q}}_{3} \leq \widehat{\mathbf{p}}_{3}^{2} \widehat{\mathbf{q}}_{4} \quad \Leftrightarrow \quad 7(1-\beta) \leq 4 .
\end{aligned}
$$

This implies that $\frac{3}{7} \leq \alpha \leq \frac{4}{7}, \frac{3}{7} \leq \beta \leq \frac{4}{7}$ and $\frac{7 \beta}{4} \leq \alpha$ and thus also that $\alpha \geq \frac{3}{4}$. As such we obtain the wanted contradiction and we conclude that there cannot exist an egoistic-CR of the data set in Example 2.

\section{Proof of Proposition 4}

Example 1 of Cherchye et al. (2007) shows that there cannot exist a general-CR of $S$ if we observe that $\mathbf{p}_{1} \mathbf{q}_{1} \geq \mathbf{p}_{1}\left(\mathbf{q}_{2}+\mathbf{q}_{3}\right), \mathbf{p}_{2} \mathbf{q}_{2} \geq \mathbf{p}_{2}\left(\mathbf{q}_{1}+\mathbf{q}_{3}\right)$ and $\mathbf{p}_{3} \mathbf{q}_{3} \geq \mathbf{p}_{3}\left(\mathbf{q}_{1}+\mathbf{q}_{2}\right)$ holds simultaneously. Without losing generality, we assume that $\mathbf{p}_{2} \mathbf{q}_{2}<\mathbf{p}_{2}\left(\mathbf{q}_{1}+\mathbf{q}_{3}\right)$.

Consider the following personalized quantities and prices for an $\alpha \in[0,1]$ :

$$
\begin{aligned}
& \widehat{\mathbf{q}}_{1}=\left(\mathbf{q}_{1}, \mathbf{0}, \mathbf{0}\right), \widehat{\mathbf{q}}_{2}=\left(\alpha \mathbf{q}_{2},(1-\alpha) \mathbf{q}_{2}, \mathbf{0}\right), \widehat{\mathbf{q}}_{3}=\left(\mathbf{0}, \mathbf{q}_{3}, \mathbf{0}\right) \\
& \mathfrak{p}_{t}^{1}=\mathbf{p}_{1}, \mathfrak{p}_{t}^{2}=\mathbf{0} \text { for } t=1,2,3 .
\end{aligned}
$$

These feasible prices and quantities are consistent with the collective model with only private goods (i.e. $\mathfrak{q}_{t}^{h}=\mathbf{0}$ ) and egoistic preferences (i.e. $\mathfrak{p}_{t}^{1}=\mathbf{p}_{t}$ and $\mathfrak{p}_{t}^{2}=\mathbf{0}$ ).

Given that $\mathbf{p}_{2} \mathbf{q}_{2}<\mathbf{p}_{2}\left(\mathbf{q}_{1}+\mathbf{q}_{3}\right)$, there must exist an $\alpha \in[0,1]$ such that $\alpha \mathbf{p}_{2} \mathbf{q}_{2}<$ $\mathbf{p}_{2} \mathbf{q}_{1}$ and $(1-\alpha) \mathbf{p}_{2} \mathbf{q}_{2}<\mathbf{p}_{2} \mathbf{q}_{3}$. One can then easily verify that for such an $\alpha$ the GARP conditions in Proposition 3 are satisfied for both members. This implies that there exists an egoistic-CR of $S$. 


\section{References}

[1] Afriat, S., 1967, "The construction of utility functions from expenditure data", International Economic Review, 8, 67-77.

[2] Browning, M., Chiappori, P.-A., 1998, "Efficient Intra-Household Allocations: A General Characterization and Empirical Tests", Econometrica, 66, 1241-1278.

[3] Cherchye, L., De Rock, B., Vermeulen, F., 2007, "The Collective Model of Household Consumption: A Nonparametric Characterization", Econometrica 75, 553574.

[4] Cherchye, L., De Rock, B., Vermeulen, F., 2010a, "An Afriat theorem for the collective model of household consumption", Journal of Economic Theory, 145, 1142-1163.

[5] Cherchye, L., De Rock, B., Vermeulen, F., 2010b, "The revealed preference approach to collective consumption behavior: nonparametric testing and sharing rule recovery", to appear in Review of Economic Studies.

[6] Chiappori, P.-A., 1988, "Rational Household Labor Supply", Econometrica, 56, 63-89.

[7] Chiappori, P.-A., 1992, "Collective Labor Supply and Welfare", Journal of Political Economy, 100, 437-467.

[8] Chiappori P.-A., Ekeland, I., 2006, "The Micro Economics of Group Behavior: General Characterization", Journal of Economic Theory, 130, 1-26.

[9] Chiappori, P.-A. and I. Ekeland, 2009, "The micro economics of efficient group behavior: identification", Econometrica, 77, 763-799.

[10] Diewert, W. E., 1973, Afriat and revealed preference theory, Review of Economic Studies, 40, 419-426.

[11] Houthakker, H. S., 1950, Revealed preference and the utility function, Economica, 17, 159-174. 
[12] Hurwicz, L., 1971, On the problem of integrability of demand functions, in J. Chipman, L. Hurwicz, M. Richter and H. Sonnenschein (eds.), Preferences, Utility, and Demand, Harcourt Brace Jovanovich, New York.

[13] Pollak, R.,1990, Distinguished Fellow: Houthakker's contributions to economics, Journal of Economic Perspectives, 4, 141-156.

[14] Samuelson, P. A., 1938, A Note on the pure theory of consumer behavior, Economica, $5,61-71$.

[15] Varian, H., 1982, "Nonparametric Approach to Demand Analysis", Econometrica, 50, 954-973. 